\title{
Internet Routing-The State of the Art
}

\author{
K.Ramasamy, A.Arokiasamy, and P.A.Balakrishnan \\ Faculty Of Engineering, Multimedia University, Malaysia \\ E-mail: k.ramasamy@mmu.edu.my
}

\begin{abstract}
The Internet protocols are the world's most popular open-system (nonproprietary) protocol suite because they can be used to communicate across any set of interconnected networks. In this paper an overview on some internet routing is analyzed. The routing protocols used in the internet are ARPANET protocol, Routing Information Protocol (RIP), Open Shortest Path First protocol (OSPF), Interior Routing Protocol (IRP), Exterior Routing Protocol (ERP), Interior Gateway Protocol (IGP), Exterior Gateway Protocol (EGP), Transfer Control Protocol (TCP) and Internet Protocol (IP). The routing protocols are used based on reliability, scalability and security. The OSPF protocol has been given the status of being the standard routing protocol for IP internetworks. The routing protocol, which is currently in use, is Transmission Control Protocol/Internet Protocol (TCP/IP), which is providing to be a great success for transmission of data. TCP provides a reliable connection for the transfer of data between applications. The Internet Protocol (IP) is a network-layer protocol that contains addressing information and source control information that enables packets to be routed. It has two primary responsibilities: providing connectionless, best effort delivery datagrams through an internetwork and providing fragmentation and ressembly of datagrams to support data links with different maximumtransmission unit (MTU) sizes. In addition to the TCP, there is another transport level protocol, the UserDatagram Protocol (UDP) which provides connectionless service for applicationlevel procedures. UDP is useful when TCP would be too complex, too slow or just unnecessary. UDP address is the combination of 32-bit IP address and 16-bit port number. Unlike IP,it does checksum its data,ensuring data integrity.IPv6 is a new version of the Internet protocol based on IPv4.It increases the IP address size from 32 bits to 128 bits to support more levels of addressing hierarchy, much greater number of addressable modes and simpler auto-configuration of address.Scalability of multicast address is introduced.A new type of address called an anycast address is also defined to send a packet to any one of a group of nodes.
\end{abstract}




\section{INTRODUCTION}

Internet is a collection of communication networks interconnected by Bridges and/or Routers. Routing is the process of finding a path from a source to every destination in the network. The heart of communication depends on routing. One such communication is internet. Here we analyze about the routing which takes place in the internet. The process of Routing in an Internetwork is referred to as Internet Routing. The principal criterion of successful routing are, correctness, computational simplicity, stability, fairness, optimality, the most reliable route and the least expensive route. In general forwarding a packet from source to destination using the best path is known as optimal routing.

A Sub network refers to a constituent network of an Internet. A device attached to one of the sub networks of an internet that is used to support end-user applications or services is called as End Systems. A device used to connect two sub networks and permit communication between end systems attached to different sub networks is known as Intermediate Systems. Two important terminologies related to Internet routing is Bridge and Router. A Bridge operates at the Data-Link layer. A Bridge is an IS used to connect two LANs that use similar LAN protocols. The bridge acts as an address filter, picking up packets from one LAN that are intended for a destination on another destination on another LAN and passing those packets on. The bridge does not modify the contents of the packets and does not add anything to the packet.

A router is a special purpose computer that transfers data to and from the next higher level of the hierarchy. Or in other words a Router is defined as a host that has an interface on more than one network. The router mainly operates on the Network layer. The key to effective performance management in a packet-based inter network environment is the router. The router must

1. Have the processing capacity to move IP data grams through the router at extremely high rates.

2. Have sufficient knowledge of the networked configuration to pick a route that is appropriate to a given class of traffic and to compensate congestion and failure in the internet work.

3. Employ a scheme for exchanging routing information with other routers that is effective and does not excessively contribute to the traffic burden.

4. Packets from one network to another network may have to be broken in to smaller pieces, this is known as segmentation. For example, Ethernet imposes a maximum packet size of 1500 bytes, and a minimum packet size of 1000 bytes is common in x.25 networks. So, a router for 
retransmission on a specific network may have to be fragmented in to smaller ones.

5. The hardware and software interfaces to various networks differ. The router must be independent of these differences.

6. The operation of the routers should not depend on an assumption of network reliability.

The ability to route traffic between the ever increasing number of networks that comprise the intemet, has become a major problem. The routers in an intemet perform much the same function as a packet-switching nodes (PSNS) in a packet-switching network. Just as the PSN is responsible for receiving and forwarding packets through a packet-switching network, the router is responsible for receiving and forwarding IP datagrams through an internet. The routers of an intemet need to make routing decisions based on knowledge of the topology and conditions of the intemet.

\section{STATE OF THE ART}

The problem of Routing in the internetworks has been studied for well over seven years. The subject has improved where a number of books have been written thoroughly examining different issues and solutions. A key distinction we will make concerning the study of routing is that between routing protocols, by which we mean mechanisms for disseminating routing information within a network and the particulars of how to use that information to forward traffic, and routing behavior, meaning how in practice the routing algorithms perform. Routing algorithms referred to as the network layer protocol that guides packets through the communication subnet to their correct destination.

Previously McQuillan has discussed about the initial ARPANET routing algorithm[1] and the algorithms that replaced it [2]; the Exterior Gateway Protocol used in the NSFNET [3] and the Border Gateway Protocol that replaced it[4]; the related work by Estrin et al., on routing between administrative domains [5]; Perlman and Verghese's discussion of difficulties in designing routing algorithms [6]; Perlman's comparison of the popular OSPF and IS-IS protocols [7]; Barasel et al's survey of routing techniques for very high speed networks [8].

Other Current works include End to End Routing behavior in the intemet by Vem Paxson [9]; Chinoy's work on Dynamics on Internet routing information [10]; Deering and Cheriton's work on Multicast Routing in Datagrarn Intemetworks and Extended LANs [11]; Stevens and Gomer's work on TCP/IP Protocols [12]. 


\subsection{Current Trends}

For Routing purpose, the Internet is partitioned in to a disjoint set of Autonomous systems (AS's), a notion first introduced in [3]. Originally an AS was a collection of routers and hosts unified by running a single "interior gateway protocol". Routing between autonomous systems provides the highest level of Internet interconnection. There are many techniques introduced after the ARPANET routing protocol. They are Routing information protocol(RIP), Open shortest path first protocol(OSPF), Interior routing protocol(IRP), Exterior routing protocol(ERP), Interior gateway protocol (IGP), Exterior gateway protocol(EGP), Transfer control protocol (TCP) and Internet protocol(IP).

All the above mentioned routing protocols are used for the purpose of intemet routing. Currently Internet protocol(IP) is used more for intemet routing on the basis of reliability, Scalability and security. We will discuss more about this in the later part.

\section{ROUTING METHODS}

There are different routing methods in existence [14]. Basic routing methods are described in the following sections.

\subsection{Source Routing}

All the information about how to get from here to there is first collected at the source, which puts it in to packets that it launches toward the destination. The job of the intervening network is simply to read the routing information from the packets and act on it faithfully.

\subsection{Hop-by-Hop Routing}

The source is not expected to have all the information about how to get from here to there ; it is sufficient for the source to know only how to get to the next hop, and for that system to know how to get to the next hop and so on until the destination is reached. The term "hop" is used to describe a unit of distance in routing algorithms.

\subsection{Direct Routing}

To see if the destination is on the same physical network:

a) Extract the NETID portion of the destination IP address. 
b) Compare with source's NETID(S)

This method is extremely efficient because of the address structure.

To send from node $\mathrm{A}$ to node $\mathrm{B}$ :

a) Encapsulate the datagram in a physical frame

b) Bind the IP address to the physical address (ARP, RARP)

c) Transmit the frame.

The final Gateway along the total route will always perform direct routing to the destination node.

\subsection{Indirect Routing}

- More difficult because it must be sent to an appropriate gateway (or default gateway).

- Gateways form a cooperative, interconnected structure which pass datagrams to each other until a gateway can deliver the datagram directly.

Indirect routing involves:

a) Software extraction of the datagrain from the frame.

b) Selecting an appropriate route based on routing algorithm used.

c) Re-encapsulation of datagram in to a new frame.

d) Setting the appropriate physical addresses in the frame header.

e) Transmit the new frame on to the network.

\subsection{Static Routing}

Static Routing is a type of routing that do not base their routing decisions on measurements or estimates of the current traffic and topology. The principle of static routing is used widely when there is an instance that routing decisions can be made independently.

\subsection{Dynamic Routing}

Dynamic routing, on the other hand, attempt to change their routing decisions to reflect changes in topology and the current traffic. The principle of dynamic routing is also used widely, because of its nature and tendency to change with the routing environment. 


\section{ROUTING PROTOCOL REVIEW}

Routing Protocols are an essential ingredient to the operation of an internet. Internets operate on the basis of routers that forward IP datagrams from router to router on a path from the source host to the destination host. For a router to perform its function, it must have some idea of the topology of the intemet and the best route to follow. It is the purpose of the Routing protocol to provide the needed information.

Routing is accomplished by means of routing protocols that establish consistent routing tables in every router in the network. Routing protocols are the base for Routing algorithms. For a routing protocol an algorithm is used called as routing algorithm.

Routing Protocols are chosen for the following criteria:

- Availability

- Speed of adaptation to finding alternate routes

- Speed in resolving routing loops

- Minimizing routing table space

- Minimizing control messages

- Overhead load placed on the network, by the protocol

- Scalability

- Security

- Robustness

\subsection{Autonomous Systems}

Routing protocols are solely based on Autonomous Systems. Generally End Systems are considered as Autonomous Systems which provides the base for routing protocols. The Autonomous System (AS) exhibits the following characteristics:

1. An AS consists of a group of routers exchanging information through a common routing protocol.

2. An AS is a set of routers and networks managed by a single organization.

3. Except in times of failure, an AS is connected (in a graph-theoretic sense).

There are two types of Routing Protocols in an Autonomous System: interior routing protocols and exterior routing protocols. 


\subsection{Interior Routing Protocol (IRP)}

The protocol which is used to pass routing information between routers within an AS is referred to as Interior routing protocols.

Most important examples of IRP is

- Routing Information Protocol (RIP)

- Open Shortest Path First Protocol (OSPF)

\subsection{Exterior Routing Protocol (ERP)}

The Protocol which is used to pass routing information between routers in different ASs is referred to as an Exterior Routing Protocol. The application of exterior and interior routing protocols is shown in figure 1.

Examples of ERP is

- Border Gateway Protocol (BGP)

- Inter Domain Routing Protocol (IDRP)

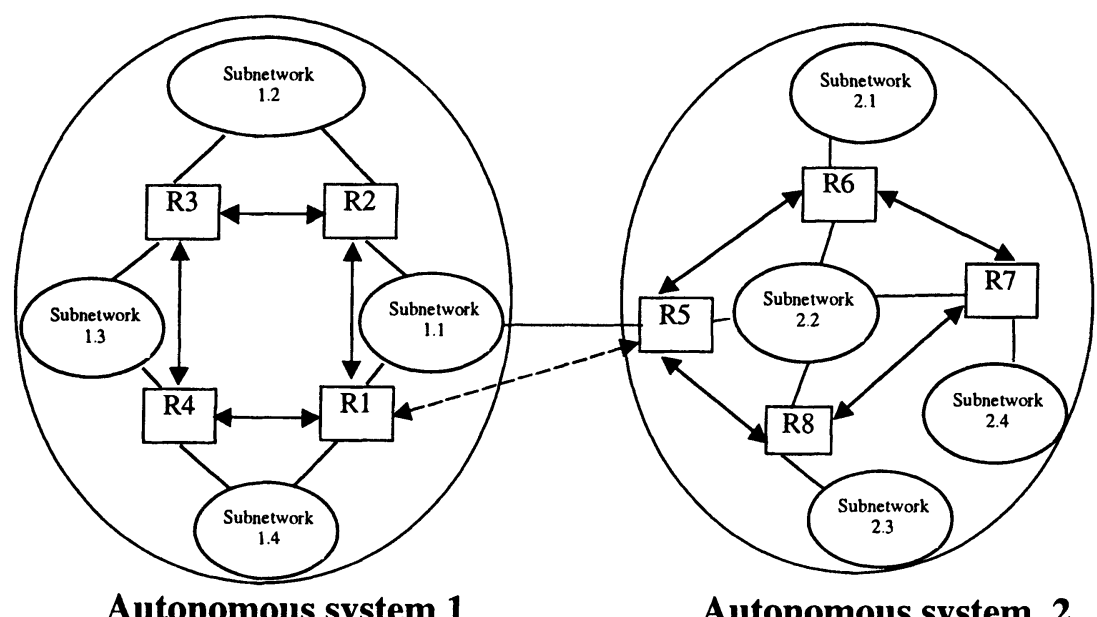

Autonomous system 1

Autonomous system 2

Figure 1: Application of exterior and interior routing protocols

\subsection{Routing Information Protocol (RIP)}

The Routing information protocol is known as distance vector protocol, which means that all of router's decisions about which path to use are based on distance The Bellman-ford algorithm is used by RIP for computing the shortest path. An example of RIP has been given. It is shown in figure 2 . 


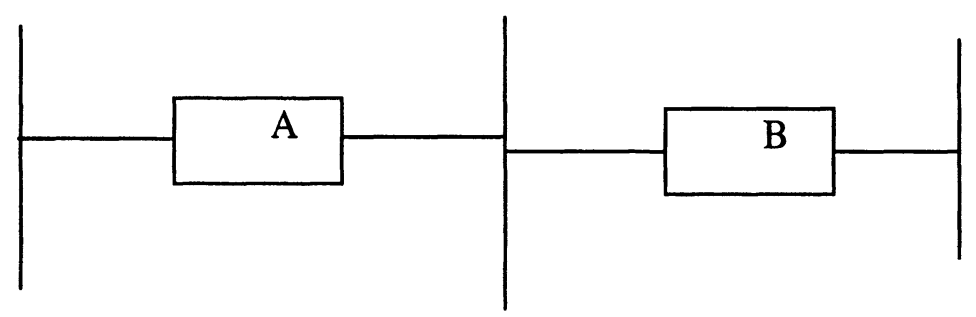

Fig.2. Basic Routing Example

Here Router A needs to compute a path to a destination that it is not directly connected to. Router A would like to get to network 3, which is on the other side of Router B. In this case where there are multiple paths to the same destination then each path will be examined and the path with the lowest cost, or number of hops, will be chosen as the path to use for that destination.

To Summarize, the cost of a path to get from A to F could be written as follows:

$$
d(A, B)+d(B, C)+d(C, D)+d(D, E)+d(E, F)
$$

Where $d(x, y)$ is the distance, or number of hops, from $x$ to $y$. In most implementations of RIP each intermediate hop is assigned a cost or metric of 1 , and if this is the case then the cost of getting from A to F is simply equal to the number of intermediate networks between the source and the destination. This formula is used in all cases of Distance Vector Routing. Generally Bellman-Ford algorithm is used for this kind of protocol. In some cases Ford-Fulkerson algorithm is also used.

A basic routing scenario is shown in figure 3 .

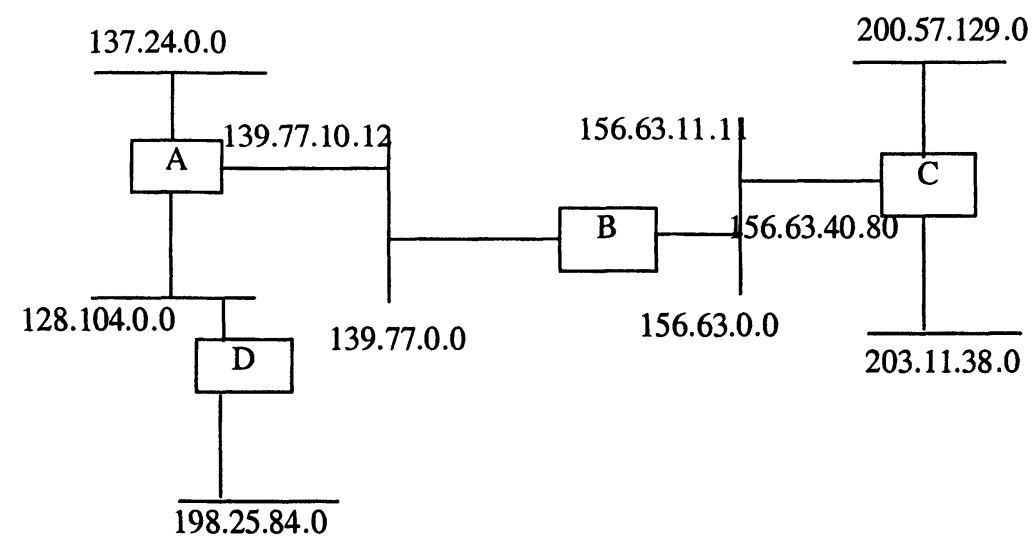

Fig.3. A basic routing scenario 


\subsubsection{Building a routing table}

In the above example we have four routers and seven networks. The initial RIP routing table is shown in table.1.

Table 1.Initial RIP routing table

Destination

Next Hop Metric D/R L/R Interface

139.77.0.0

0.0.0.0

I

D $\quad \mathrm{L} \quad 1$

156.63.0.0

0.0.0.0

D $\mathrm{L} \quad 2$

Here, Destination is the Destination network number. Next hop lists the complete IP address of the next hop router that is between you and the destination network. Metric is the hop count field, it lists how far away the particular destination is from you.

D/R: D Directly connected network; $\mathrm{R}=$ Remote network.

L/R: L Network was learned because it is local.

Interface is the logical interface number on which the routing information was received.

The completed RIP Routing Table is shown in table 2.

Table 2. Completed RIP Routing Table

Destination

128.104.0.0

137.24.0.0

139.77.0.0

156.63.0.0

198.25.84.0

200.57.129.0

203.11.38.0
Next Hop

139.77.10.12

139.77.10.12

0.0.0.0

0.0.0.0

139.77.10.12

156.63.40.80

156.63.40.80
Metric Interface

2

2

$1 \quad 1$

12

$3 \quad 1$

22

22

\subsubsection{RIP Message Format}

The RIP message format is shown in figure 4.

\begin{tabular}{|c|}
\hline Command (8) \\
\hline Version (8) \\
\hline Must be zero (16) \\
\hline Address Family Identifier (16) \\
\hline Must be zero (16) \\
\hline IP Address (32) \\
\hline Must be zero (64) \\
\hline Metric (32) \\
\hline
\end{tabular}

Fig.4. RIP message format 
The Description of RIP message format is given as follows

Command:

Version:

Address Family Identifier:

IP Address :

Metric:
Defines whether this RIP message is a request (1) or a response (2).

The version of RIP being used. Current version of RIP being used is 1 .

Identifier of the protocol that is using RIP for its routing Table calculations. The Address family identifier for IP is 2 .

This is the IP address of the network that is being advertised or requested. This value may be just a network, or it may be full IP address.

The number of hops to the destination network. This value will be between 1 and 15 , with the value 16 used to set the metric to infinity.

\subsubsection{RIP Limitations}

1. Limited Network Diameter: This refers to the limited hop count allowed by RIP. Without the use of extended RIP (ERIP), your network design is limited to a maximum of fifteen routers between any two networks.

2. Convergence: The time it takes to learn about changes in the network can cause a number of problems because routers believe erroneous information longer than they should.

3. Aging of Entries: Entries in a routing table are allowed to remain in the routing table for 180 seconds in the absence of an update about the network.

4. Subnetting: It is important to remember that RIP assumes that the same subnet mask is being used throughout the entire RIP environment.

\subsection{Open Shortest Path first Protocol (OSPF)}

The Open shortest path first protocol has been given the status of being the standard routing protocol for IP internetworks. OSPF was created specifically for the IP environment to solve many problems inherent with RIP. Here the Algorithm used is Dijkstra's algorithm for finding the shortest path. A "path" is a series of steps needed to get from one point to another point in the intemetwork.

OSPF uses the concept of areas and the backbone to allow us to divide the domain in to manageable entities. The procedure of getting from one area to another can be broken up in to three steps:

1. The path from the source network to the edge of the originating area. 
2. The path from the originating area, across the backbone, to the edge of the destination area.

3. The path from the edge of the destination area to the destination network.

\subsubsection{Shortest Path Tree}

The Shortest path tree shows the best path to each destination in the intemetwork with the associated total path cost. It is from the shortest path tree that we build a routing table. The shortest path tree for router11 is given as example, which is as shown in figure 5.

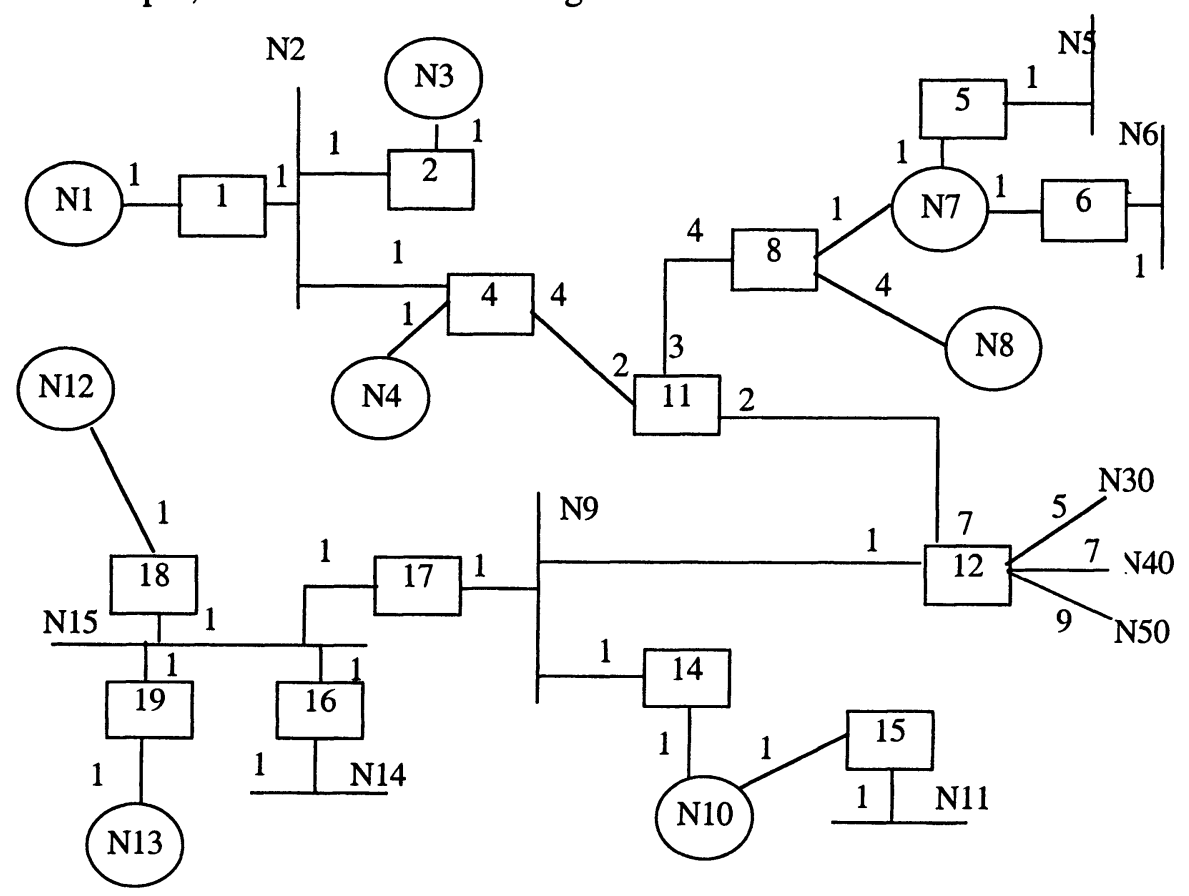

Fig.5. Shortest path tree for router11

A specific example has been given to show the shortest path area. The corresponding basic routing table is shown in table. 3 .

Dijkstra's algorithm is a graph theory algorithm that has been applied to the routing world, specifically to OSPF. A sample Dijkstra's algorithm graph is shown in figure 6. 
Table 3. Corresponding basic routing table

$\begin{array}{lll}\text { Destination } & \text { Next Hop } & \text { Cost } \\ \text { NI } & \text { Router 4 } & 4 \\ \text { N2 } & \text { Router 4 } & 3 \\ \text { N3 } & \text { Router 4 } & 4 \\ \text { N4 } & \text { Router 4 } & 3 \\ \text { N5 } & \text { Router 8 } & 5 \\ \text { N6 } & \text { Router 8 } & 5 \\ \text { N7 } & \text { Router 8 } & 4 \\ \text { N8 } & \text { Router 8 } & 4 \\ \text { N9 } & \text { Router 12 } & 3 \\ \text { N10 } & \text { Router 12 } & 4 \\ \text { N11 } & \text { Router 12 } & 5 \\ \text { N12 } & \text { Router 12 } & 5 \\ \text { N13 } & \text { Router 12 } & 5 \\ \text { N14 } & \text { Router 12 } & 5 \\ \text { N15 } & \text { Router 12 } & 4 \\ \text { N30 } & \text { Router 12 } & 5 \\ \text { N40 } & \text { Router 12 } & 7 \\ \text { N50 } & \text { Router 12 } & 9\end{array}$

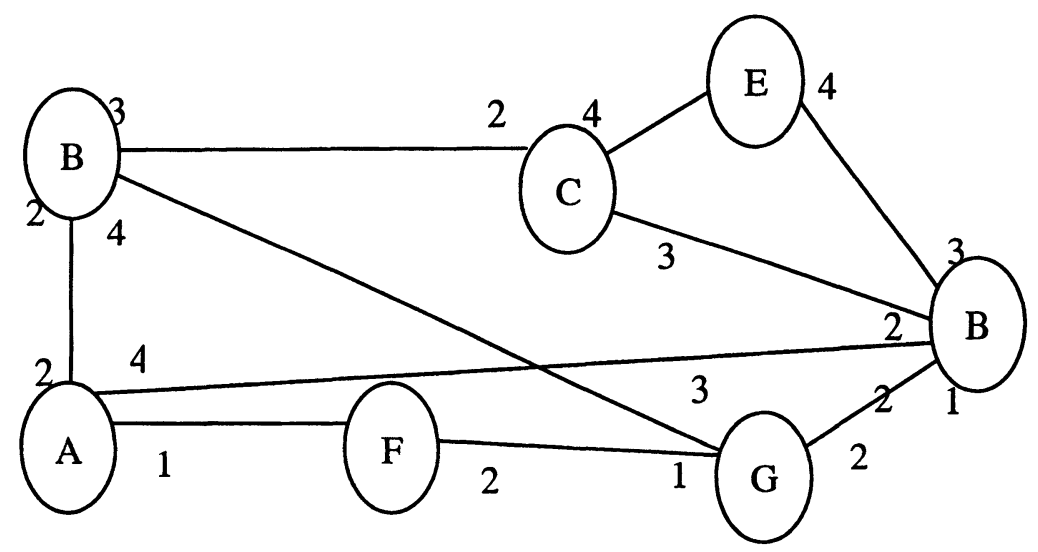

Fig.6. A sample Dijkstra's algorithm graph

The cost of a path between the source(S) and the destination(T) is:

$$
d(S, N 1)+d(N 1, N 2)+d(N 2, N 3) \ldots+d(N x, T)
$$

Where $d(x, y)$ is the cost to get from point $x$ to point $y$ on the graph. The calculation is done based on the given formula. 


\subsubsection{Router Classifications:}

The Router classifications for the OSPF is given with the illustrations. The OSPF defines four types of routers, they are as follows;

Internal Routers:

Area Border Routers:

Backbone Routers:

Autonomous System

Boundary Routers:
An Internal router is a router that has all of its interfaces connected to networks within the same area.

An area border router is a router that has network connections to more than one area.

A backbone router is a router that has interfaces connected to the backbone.

Autonomous System Boundary routers are routers that have interfaces attached to network outside autonomous system.

The RIP is generally called as Distance vector Routing and the OSPF is generally called as Link state Routing. A comparison is made between the two important routing algorithms. It is shown in table. 4.

Table.4. Comparison is made between DVR and LSR

\begin{tabular}{|l|l|}
\hline \multicolumn{1}{|c|}{ Distance-Vector Routing } \\
\hline $\begin{array}{l}\text { Each router sends routing information } \\
\text { to its neighbors }\end{array}$ & $\begin{array}{l}\text { Each router sends routing } \\
\text { information to all other } \\
\text { routers }\end{array}$ \\
\hline $\begin{array}{l}\text { The information sent is an estimate of } \\
\text { its Path cost to all networks }\end{array}$ & $\begin{array}{l}\text { The information sent is the } \\
\text { exact value of its link cost to } \\
\text { adjacent networks }\end{array}$ \\
\hline $\begin{array}{l}\text { Information is sent on a regular } \\
\text { periodic Basis }\end{array}$ & $\begin{array}{l}\text { Information is sent when } \\
\text { changes occur }\end{array}$ \\
\hline $\begin{array}{l}\text { A router determines next-hop } \\
\text { information by using the distributed } \\
\text { Bellman-Ford algorithm on the } \\
\text { received estimated path costs }\end{array}$ & $\begin{array}{l}\text { A router first builds up a } \\
\text { description of the topology of } \\
\text { the internet and then may use } \\
\text { any routing algorithm to } \\
\text { determine next-hop } \\
\text { information }\end{array}$ \\
\hline
\end{tabular}

\section{LATEST ROUTING PROTOCOL}

The Routing protocol which is currently in use is Transmission Control Protocol/ Internet Protocol (TCP/IP) [13]. This is proving to be a great 
success for transmission of data. TCP/IP is a result of protocol research and development conducted on the experimental packet-switched network, ARPANET, funded by the Defense Advanced Research Projects Agency (DARPA), and is generally referred to as the TCP/IP protocol suite. There is no official protocol model for TCP/IP like OSI. But based on the protocol standards, we can organize the communication task for TCP/IP into five relatively independent layers:

- Application layer

- Host-to-host, or Transport layer

- Internet layer

- Network access layer

- Physical layer.

\subsection{TCP and UDP}

For most applications running as part of the TCP/IP protocol architecture, the transmission layer protocol is TCP. TCP provides a reliable connection for the transfer of data between applications [13]. The figure.7. shows the header format for TCP;

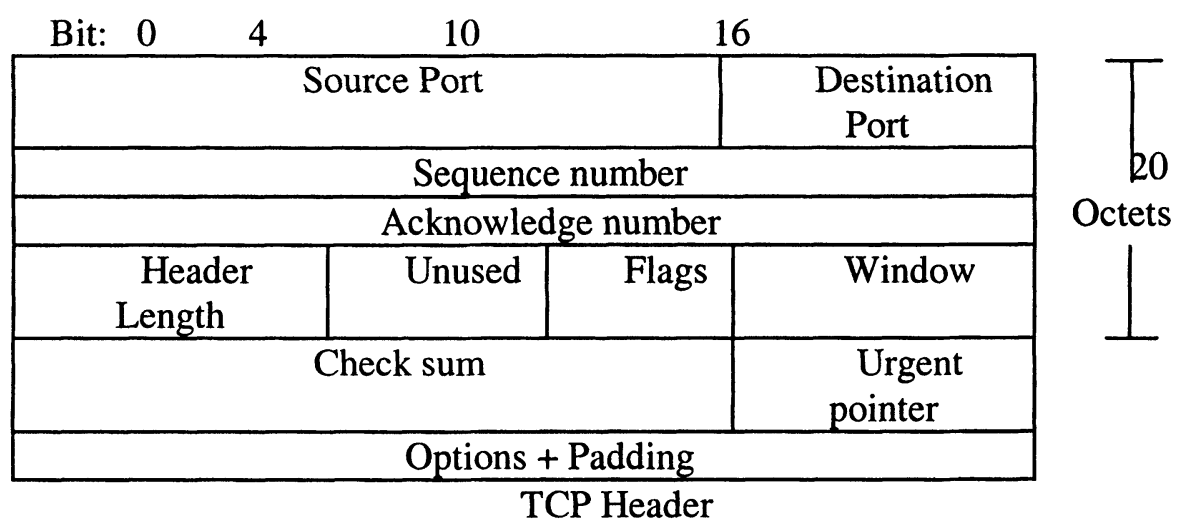

Fig.7. Header format for TCP

The header format for TCP, which is a maximum of 20 octets, or 160 bits. The source port and destination port fields identify the applications at the source and the destination systems that are using this connection. The sequence number, Acknowledgement Number, and window fields provide flow control and error control. 
In addition to the TCP, there is another transport level protocol : the user datagram protocol (UDP). UDP provides a connectionless service for applicationlevel procedures. The UDP header is shown in figure 8 .

\begin{tabular}{|l|l|} 
Bits: 0 & 32 \\
\hline Source Port & Destination Port \\
\hline Segment length & Check sum \\
\hline
\end{tabular}$\quad \begin{gathered}8 \\
\text { Fig.8. UDP Header }\end{gathered}$

UDP enables a procedure to send messages to other procedures with a minimum of protocol mechanism.

\subsection{1 lpv4 and lpv6}

The keystone of the TCP/IP protocol architecture has been the IP [13] The figure 9 shows the lpv4 header format.

\begin{tabular}{|c|c|c|c|c|} 
Bits: 0 & \multicolumn{1}{c}{4} & 16 & 19 \\
\hline Version & IHL & $\begin{array}{c}\text { Type of } \\
\text { service }\end{array}$ & \multicolumn{2}{c|}{ Total Length } \\
\hline Identification & Flags & $\begin{array}{l}\text { Fragment } \\
\text { Offset }\end{array}$ \\
\hline \multicolumn{3}{|c|}{ Protocol } & Header Checksum \\
\hline Time to live & Source address \\
\hline \multicolumn{3}{|c|}{ Destination address } \\
\hline & Options + Padding \\
\hline & Fig.9. lpv4 header format
\end{tabular}

The above IP header format, which is a minimum of 20 octets, or 160 bits. The header includes 32-bit source and destination addresses. The protocol field indicates whether TCP, UDP, or some other higher-layer protocol is using IP. The figure 10. shows the header format of the latest lpv6 protocol.

The latest Ipv6 contains 40 octets, or 320 bits. There are lot of modifications has been done to improvise the latest version. lpv6 includes 128-bit source and destination address fields. 


\begin{tabular}{|l|l|l|l|} 
Bits: $0 \quad 4 \quad 16$ & 24 & 31 \\
\hline Version & Priority & \multicolumn{3}{|c|}{ Flow Label } \\
\hline \multicolumn{5}{|c|}{ Pay load length } & Next header & $\begin{array}{l}\text { Hop } \\
\text { limit }\end{array}$ \\
\hline \multicolumn{4}{c}{ Source address } \\
\hline \\
Destination address
\end{tabular}

Fig.10. Header format of the latest lpv6 protocol

\subsubsection{Operation of TCP/IP}

Figure 11 shows the concepts of TCP/IP [13]. It also indicates how these protocols are configured for communications.

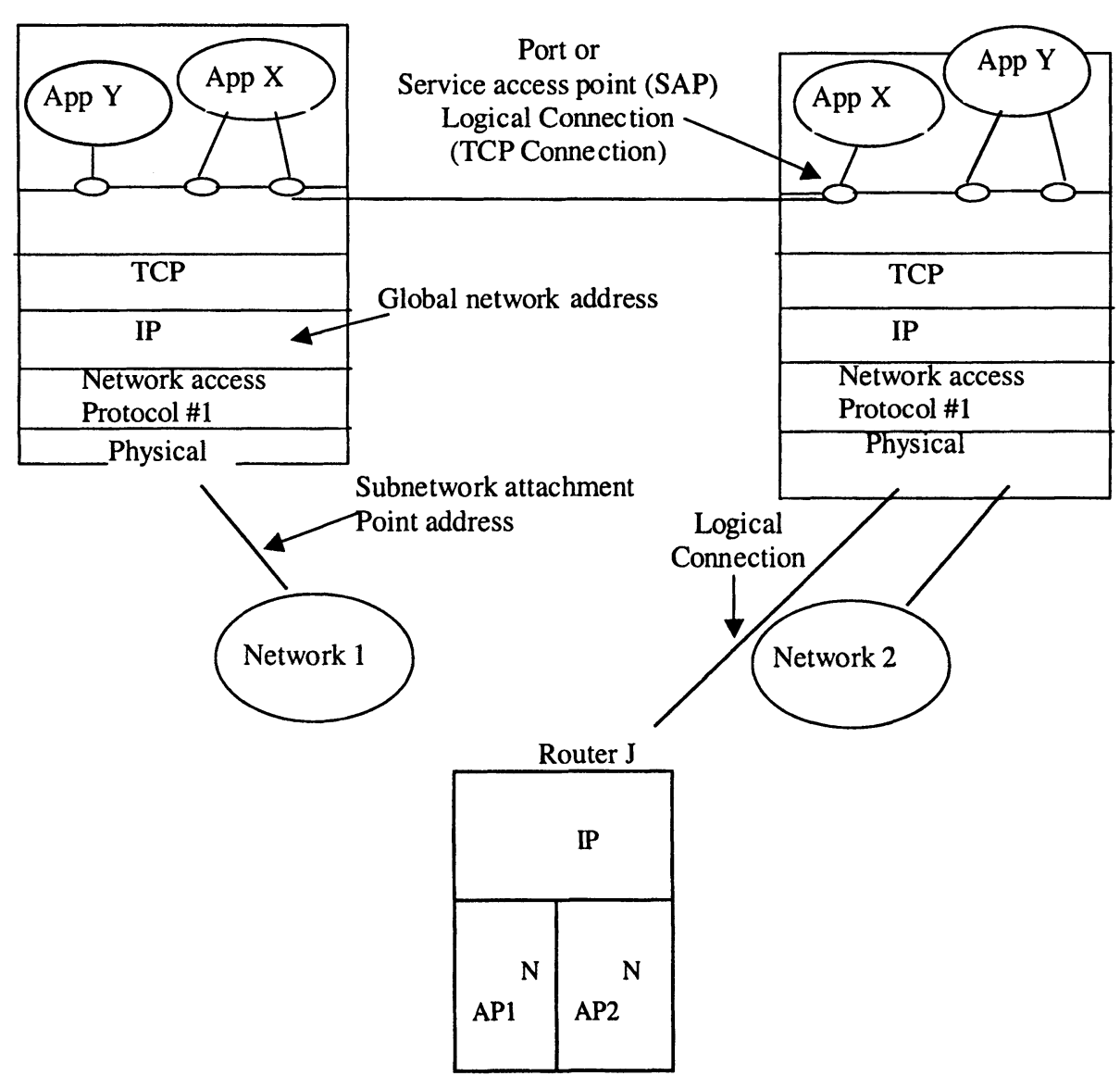

Fig.11. Basic concepts of TCP/IP 
This Protocol enables the host to send data across the subnetwork to another host, in the case of a host on another subnetwork, to a router. IP is implemented in all end systems and routers. It acts as a relay to move a block of data from one host, through one or more routers, to another host. TCP is implemented only in the end systems; it keeps track of the blocks of data to assure that all are delivered reliably to the appropriate application. For successful communication, every entity in the overall system must have a unique address.

The protocol data units in the TCP/IP architecture is shown in figure 12 .

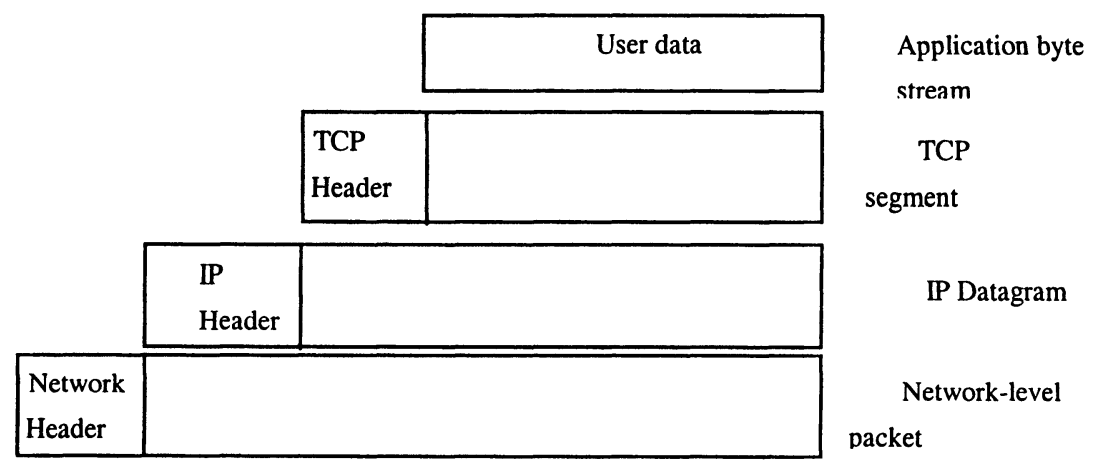

Fig.12. Protocol data units in the TCP/IP architecture

Here there are four important levels. The base most level is associated with the Network layer. The next level is associated with the Internet layer. The third level is associated with the Transport layer. The final level is associated with the Application layer. The TCP/IP architecture entirely depends on these four levels. The data communication takes place only with the cooperation of these four levels. To control the operation of TCP/IP , control information as well as user data must be transmitted. Let us assume that the sending process generates a block of data and passes this to TCP. TCP may break this block into smaller pieces to make it manageable. To each of these pieces, TCP appends control information in the TCP header, forming a TCP segment. The control information is to be used by the TCP protocol entity at host $\mathrm{B}$.

\subsection{Comparison with Previous Protocol}

After the analysis of TCP/IP we can clearly see the advantages over OSI. A comparison of the TCP/IP and OSI protocol is shown in figure 13. 


\begin{tabular}{|c|c|}
\hline Presentation & \multirow{3}{*}{$\begin{array}{l}\text { Annlication } \\
\text { Transport } \\
\text { (host-to-host) }\end{array}$} \\
\hline session & \\
\hline Transport & \\
\hline Network & Internet \\
\hline Data link & $\begin{array}{l}\text { Network } \\
\text { access }\end{array}$ \\
\hline Physical & Physical \\
\hline
\end{tabular}

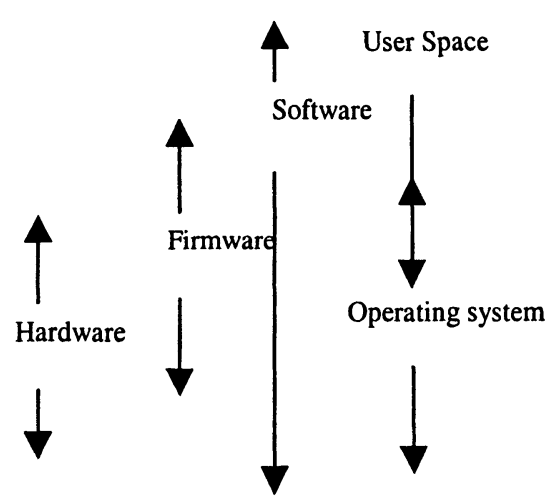

Fig.13. Comparison of the TCP/IP and OSI protocol

The Overall Operation of TCP/IP, that is its Action at Sender, Action at Router and its Action at Receiver is shown in a brief way to see the exact process.

The figure 14 shows about Action at Sender[13];

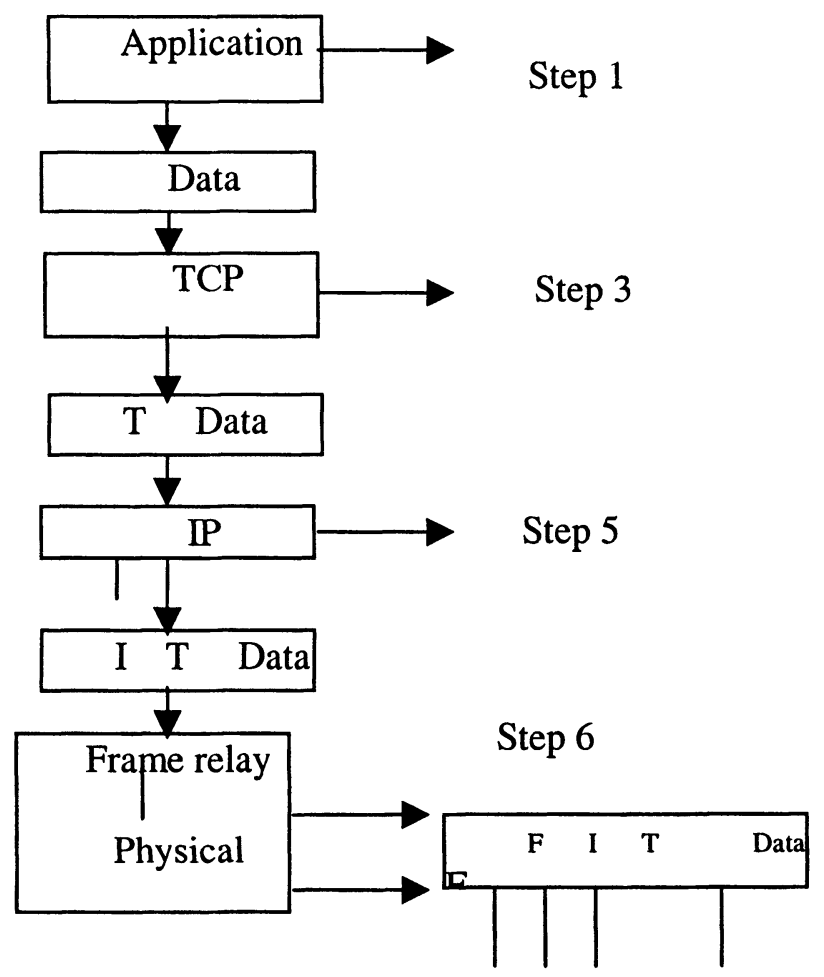

Fig.14.The Action at Sender 
The operation of TCP/IP action at sender is described in the following steps with peer-to-peer dialogue;

Step 1: Preparing the data: The application protocol prepares a block of data for transmission. For example, an e-mail message (SMTP), a file (FTP), or a block of user input (TELNET).

Peer-to-peer dialogue: Before data are sent, the sending and receiving applications agree on format and encoding and agree to exchange data.

Step 2: Using a common syntax : If necessary, the data are converted to a form expected by the destination. This may include a different character code, the use of encryption, and/or compression.

Step 3: Segmenting the data: TCP may break the data block into a number of segments, keeping track of their sequence. Each TCP segment includes a header containing a sequence number and a frame check sequence to detect errors. Peer-to-peer dialogue: The two TCP entities agree to open a connection.

Step 4: Duplicating segments: A copy is made of each TCP segment, in case the loss or damage of a segment necessities retransmission. When an acknowledgement is received from the other TCP entity, a segment is erased.

Step 5: Fragmenting the segments: IP may break a TCP segment into a number of datagrams to meet size requirements of the intervening networks. Each datagram includes a header containing a destination address, a frame check sequence, and other control information.

Peer-to-peer dialogue: Each IP datagram is forwarded through networks and routers to the destination system.

Step 6: Framing: A frame relay header and trailer is added to each IP datagram. The header contains a connection identifier and the trailer contains a frame check sequence.

Peer-to-peer dialogue: Each frame is forwarded through the frame relay network.

Step 7: Transmission: Each frame is transmitted over the medium as a sequence of bits. The Action at router[13] is clearly analyzed in the following figure. 15 . 


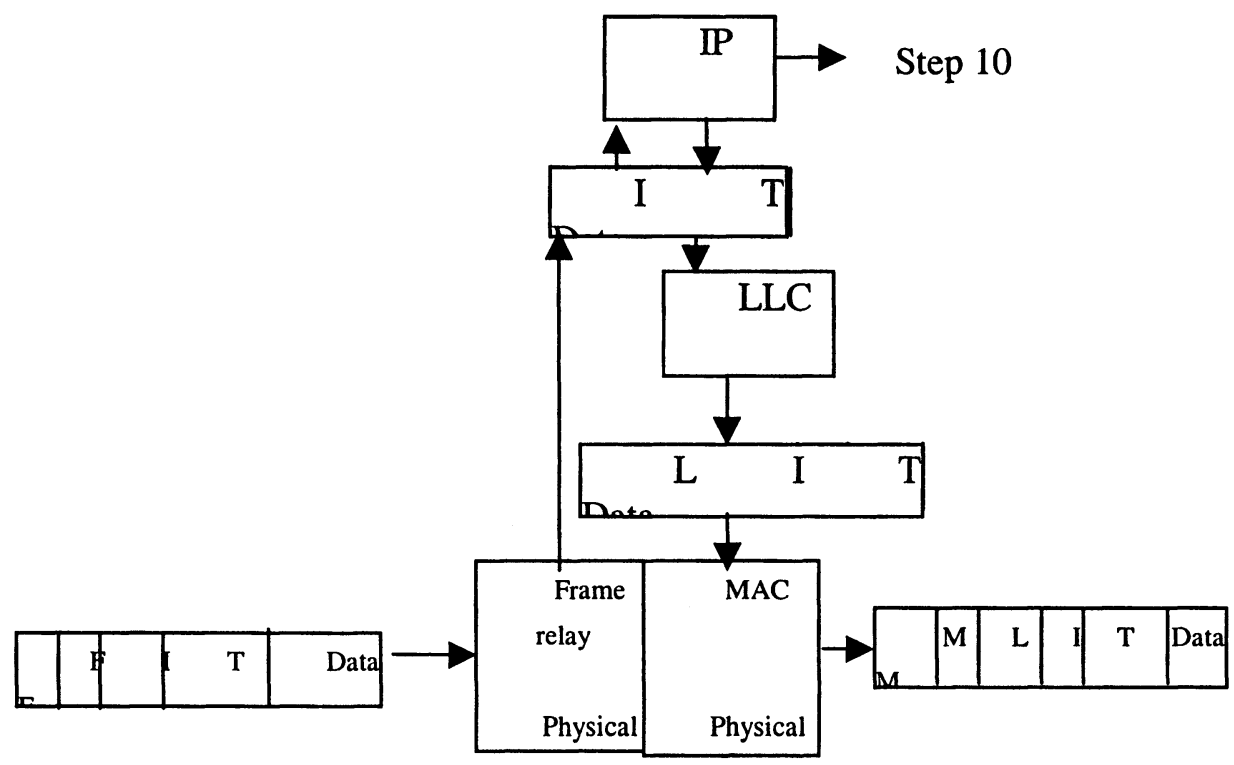

Fig.15. The Action at router

The operation of TCP/IP Action at router is described in the following steps;

Step 8: Arriving at router: The incoming signal is received over the transmission medium and interpreted as a frame of bits.

Step 9: Processing the frame: The frame relay removes the header and trailer and processes them. The frame check sequence is used for error detection. The connection number identifies the source.

Step 10: Routing the Packet: IP examines the IP header and makes a routing decision. It determines which outgoing link is to be used and then passes the datagram back to the link layer for transmission on that link.

Peer-to-peer dialogue: The router will pass this datagram onto another router or to the destination system.

Step 11: Forming LLC PDU: An LLC header is added to each IP datagram to form an LLC PDU. The header contains sequence number and address information.

Step 12: Framing: A MAC header and trailer is added to each LLC PDU, forming a MAC frame. The header contains address information and the trailer contains a frame check sequence. 
Step 13: Transmission: Each frame is transmitted over the medium as a sequence of bits.

The Action at Receiver [13] is shown in figure 16.

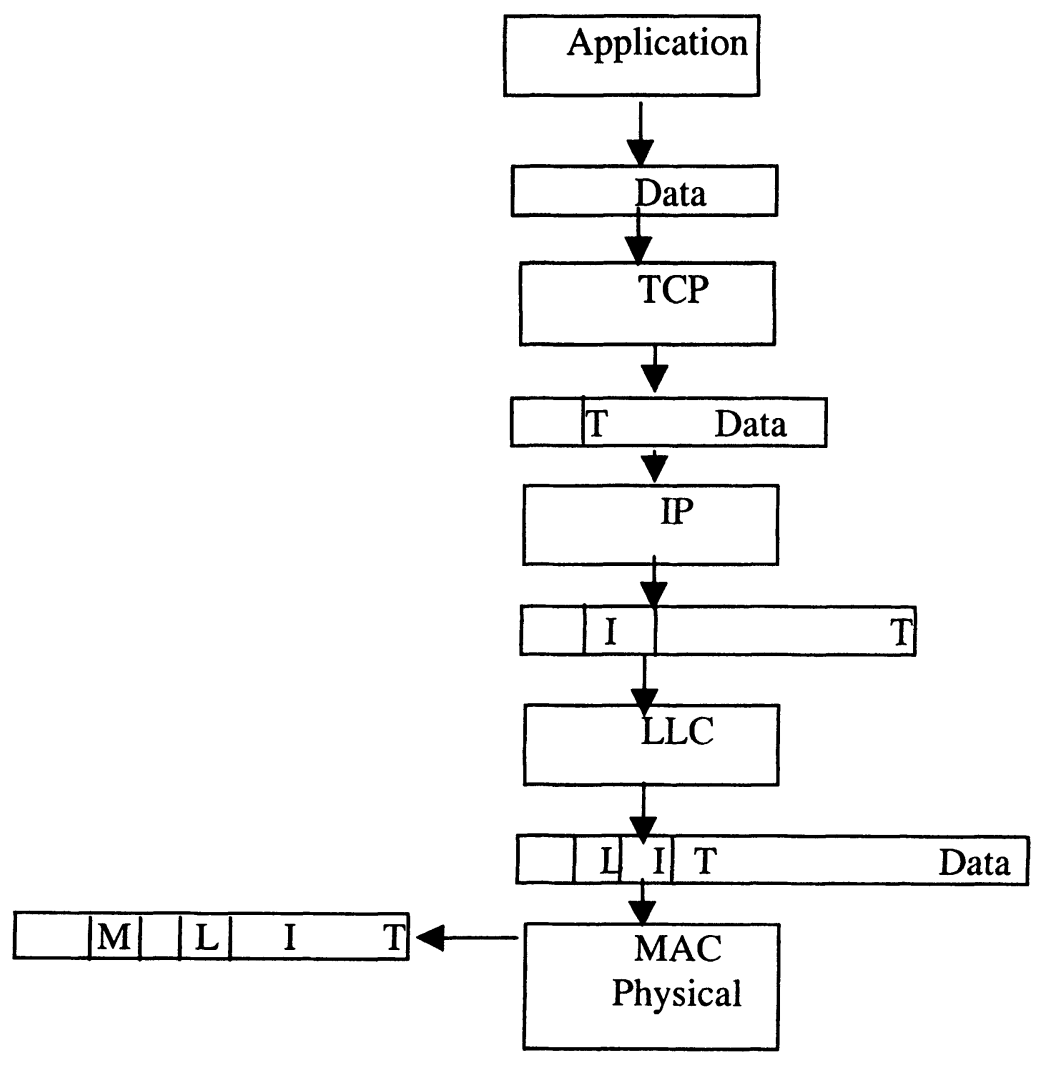

Fig.16. The Action at Receiver

The operation of TCP/IP action at receiver is described in the following steps;

Step 14: Arriving at destination: The incoming signal is received over the transmission medium and interpreted as a frame of bits.

Step 15: Processing the frame: The MAC layer removes the header and trailer and processes them. The frame check sequence is used for error detection.

Step 16: Processing the frame: The LLC layer removes the header and processes it. The sequence number is used for flow and error control. 
Step 17: Processing the IP datagram: IP removes the header. The frame check sequence and the other control information are processed.

Step 18: Processing the TCP segment: TCP removes the header. It checks the frame check sequence and acknowledges if there is a match and discards for mismatch. Flow control is also performed.

Step 19: Reassembling user data: If TCP has broken the user data into multiple segments, these are reassembled and the block is passed up to the application.

Step 20: Delivering the data: The application performs the needed transformations, including decompression and decryption, and directs the data to the appropriate file or other destination.

\section{OVERVIEW OF ROUTING PROTOCOLS}

The overall comparison of some Routing Protocols and its characteristics are shown in the following table.5.

\section{CONCLUSION}

The Routing in the Internet is clearly one of the important problems faced during this era. There are some few important routing algorithms that essentially fit for internet routing. Still there are some more algorithms which can ease the problems of intemet routing. Global information, by its very nature, is hard to collect, subject to frequent change, and voluminous. How can we summarize this information to extract only the portions relevant to each node, this lies in the heart of Routing Protocols.

We conclude that any routing protocol must communicate global topological information to each routing element to allow it to make local routing decisions. Global Optimization is very much possible through Internet Routing. This is possible only based on the routing protocols.

\section{FUTURE WORK}

There are some Efficient Loop free routing algorithm reported to date and perhaps to find the shortest path. A problem that needs to be investigated is how to ensure that routers using shortest path will never 
update their routing information using stale distance vectors. Other interests could be to simulate data traffic and measure the resulting packet loss from looping.

Table.5.Overall comparison of some Routing Protocols and its characteristics

\begin{tabular}{|c|c|c|c|c|c|c|c|c|}
\hline \multirow{2}{*}{$\begin{array}{c}\text { Character- } \\
\text { istics } \\
\end{array}$} & & & \multicolumn{3}{|c|}{ Routing Protocols } & \multirow[b]{2}{*}{ BGP } & \multirow[b]{2}{*}{ RTMP } & \multirow[b]{2}{*}{ DEC net } \\
\hline & RIP & IGRP & OSPF & $\begin{array}{l}\text { IS-IS } \\
\text { ISO } \\
\text { ES-IS }\end{array}$ & EGP & & & \\
\hline $\begin{array}{l}\text { Static Vs. } \\
\text { Dynamic }\end{array}$ & D & D & D & D & D & D & D & $\mathrm{D}$ \\
\hline $\begin{array}{c}\text { Distributed Vs. } \\
\text { Centralized }\end{array}$ & D & D & D & D & D & $\mathrm{D}$ & D & D \\
\hline $\begin{array}{l}\text { Single Vs. } \\
\text { Multi-path }\end{array}$ & $\mathbf{S}$ & $\mathbf{M}$ & $\mathbf{M}$ & $\begin{array}{c}\text { ES-IS=SS } \\
\text { IS-IS=M }\end{array}$ & M & $S$ & $\mathbf{M}$ & \\
\hline $\begin{array}{c}\text { Flat Vs. } \\
\text { Hierarchical }\end{array}$ & $\mathbf{F}$ & F & $\mathrm{H}$ & $\begin{array}{l}E S-I S=F \\
I S-I S=H\end{array}$ & F & F & $\mathrm{F}$ & $\mathrm{H}$ \\
\hline $\begin{array}{c}\text { Source Vs. } \\
\text { Router } \\
\text { Intelligent }\end{array}$ & $\mathbf{R}$ & $\mathrm{R}$ & $\mathbf{R}$ & $\mathrm{R}$ & $\mathbf{R}$ & $\mathbf{R}$ & $\mathbf{R}$ & $\mathrm{R}$ \\
\hline \begin{tabular}{|c|} 
Intra- Vs. \\
Inter-domain \\
\end{tabular} & Intra & Intra & Intra & $\begin{array}{l}\text { ES-IS = Intra } \\
\text { IS-IS= both }\end{array}$ & Inter & Inter & Inter & Both \\
\hline $\begin{array}{c}\text { Link state Vs. } \\
\text { Distance } \\
\text { Vector } \\
\end{array}$ & DV & DV & IS - IS =LS & $\begin{array}{l}\text { ES -IS = } \\
\text { Neither }\end{array}$ & $\begin{array}{l}\text { Neither } \\
\text { (Reach- } \\
\text { ability) }\end{array}$ & $\begin{array}{l}\text { Neither } \\
\text { (Reach- } \\
\text { ability) }\end{array}$ & DV & LS \\
\hline $\begin{array}{c}\text { Yrs. Since } \\
\text { Intial } \\
\text { Specification }\end{array}$ & 10 & 6 & 2 & 5 & 9 & 2 & 7 & 12 \\
\hline $\begin{array}{c}\text { Metric } \\
\text { Factors } \\
\text { considered }\end{array}$ & $\begin{array}{l}\text { Hop-count } \\
\text { Delay } \\
\text { Bandwidth } \\
\text { Load MTU } \\
\text { Range: } \\
\text { 1-16 }\end{array}$ & $\begin{array}{c}\text { Reliability Unit } \\
\text { Range: } 1-255 . \\
\text { Number } \\
\text { Optional TOS } \\
\text { Service Cost }\end{array}$ & $\begin{array}{l}\text { Arbitrary IS- } \\
\text { IS=Arbitrary } \\
\text { Unit Number } \\
\text { Range: } \\
\text { 1-65536 }\end{array}$ & $\begin{array}{c}\text { ES-IS }=\ldots . \\
\text { Range: } \\
1-1024\end{array}$ & $\begin{array}{c}\text { Unit } \\
\text { Number }\end{array}$ & Arbi-trary & $\begin{array}{c}\text { Hop-count } \\
\text { Unit } \\
\text { Number } \\
\text { Range: } \\
1-16\end{array}$ & $\begin{array}{c}\text { Arbi- } \\
\text { trary } \\
\text { Range } \\
1-1022\end{array}$ \\
\hline
\end{tabular}

Because of the growing size of the internetworks, it would be of great interest to extend shortest path algorithms to hierarchical networks. A promising approach to address this problem is to adopt McQuillan's scheme to hierarchical routing. Some simulation results of hierarchical routing scheme should outperform OSPF. 
Research on Internet Routing to transmit Audio and Video is well in progress. In future the study of routing in streams should be done by minimizing the delays in the frame relays.

\section{REFERENCES}

[1] J.McQuillan, G.Falk and I.Ritcher, " A Review of the development and Performance of the ARPANET Routing Algorithm", IEEE Transactions on Communications, 26(12), pp.1802-181 1, December 1978.

[2] J.McQuillan, I.Ritcher and E.Rosen, "The New routing algorithm for the ARPANET", IEEE Transactions on communcations, 28(5), pp.711-719, May 1980.

[3] E.Rosen, "Exterior Gateway Protocol (EGP)", RFC 896, Network Information Center, SRI International, Menlo Park, CA, October 1982.

[4] Y. Rekhter, "Inter - Domain Routing protocol: EGP, BGP, and IDRP", in Prentice hall, 1995 .

[5] L.Breslau and D.Estrin, "Design of Inter-Administrative Domain routing protocols", Proceedings of SIGCOMM' 90, pp. 231-241, September 1990.

[6] R.Perlman and G.Varghese, "Pitfalls in the Design of Distributed routing algorithms", Proceediings of SIGCOMM' 88, pp. 43-54, August 1988.

[7] R.Perlman, "A comparison between two routing protocols: OSPF and IS-IS", IEEE Network, 5(5), pp. 18-24, September 1991.

[8] C.Baransel, W.Dobosiewicz, and P.Gburzynski, "Routing in multihop packet switching networks", IEEE Network, 9(3), pp. 38-61, May/June 1995.

[9] Vem Paxson, "End to End Routing behavior in the intemet", Lawrence Berkeley National Laboratory, University of California, Berkeley, May 1996.

[10] B.Chinoy, "Dynamics of Internet routing information", Proceedings of SIGCOMM' 93, pp.45-52, September 1993.

[11] S. Deering and D. Cheriton, "Multicast Routing in datagram internetworks and extended LANS", ACM Transactions on Computer Systems, 8 (2), pp. 85 - 110, May 1990.

[12] W.R.Stevens and Comer, TCP/IP Illustrated, volume 1: The Protocols, Addison Wesley, 1994.

[13] William Stallings, High Speed Networks - TCP/IP and ATM design Principles,Prentice hall, 1998.

[14] S.Keshav, An Engineering approach to Computer Networking - ATM networks, the intemet, Addison wesley, 1997.

[15] Mark Dickie, Routing in today's Internetworks, VNR Communications laboratory,1993.

[16] John D. Spragins with Joseph L. Hammond and Krzysztof Pawlikowski, Telecommunications Protocols and Design, Addison-Wesley, July 1992.

[17] Christian Huitema, Routing in the Internet, Prentice - Hall, 1995. 